\title{
Optical method for inspecting surface defects inside a small bore
}

\author{
En Hong ${ }^{1}$, Reuven Katz ${ }^{1}$, Brian Hufnagel ${ }^{1}$ and John Agapiou ${ }^{2}$ \\ ${ }^{1}$ Engineering Research Center/Reconfigurable Manufacturing Systems, Mechanical Department, \\ University of Michigan, Ann Arbor, MI, USA \\ ${ }^{2}$ General Motors Research and Development Center, Warren, MI, USA \\ E-mail: enhong@umich.edu
}

Received 16 July 2009, in final form 30 October 2009

Published 7 December 2009

Online at stacks.iop.org/MST/21/015704

\begin{abstract}
Most automotive powertrain parts made of castings have surface defects such as pores. However, detecting pores inside small diameter bores is a challenge because of the limited dimensional accessibility. Adding to this difficulty is the auto industry's desire to conduct the porosity inspection in-line, i.e. within the machining production cycle time of a part. A technique or equipment that meets these requirements currently does not exist. In order to meet these demands, it is necessary to develop an entire new methodology to inspect the inner surface of small diameter bores. This paper presents an innovative methodology to inspect the porosity of the inner surface of small bores and to provide their characteristics such as size and location. A prototype measurement system was built and tested in the lab. Experimental results showed the proposed method to be reliable and consistent.
\end{abstract}

Keywords: porosity, sight-pipe, line-scan, position-synchronization-output, quality control, reconfigurable inspection system

(Some figures in this article are in colour only in the electronic version)

\section{Introduction}

Porosity is one of the most frequently found defects in castings. Surface porosity is caused by tiny voids or pits at the surface of the machined castings. This problem begins during the casting process when gas is trapped in the metal, creating voids in the material. If subsequent machining exposes these voids, small pits will be left on the surface. Although they are typically smaller than $1 \mathrm{~mm}$, surface pores can cause significant leaks of coolant, oil and combustion gases between critical mating surfaces and cause severe damage to powertrain components such as engine cylinder head and transmission valve. Thus, the occurrence of pores (voids) at the surface of machined castings is a particular concern among manufacturers of powertrain components.

Due to the advancing of the casting techniques the current auto industry is able to produce more and more complicated and accurate parts which require less machining. A good example of such parts is a transmission valve body. These valve bodies have multiple small bores with diameter from $8 \mathrm{~mm}$ to $20 \mathrm{~mm}$. These bores have a huge number of features because of the designing discontinuity and steps. This surface complicacy will cause a higher occurrence of pore and make pore detection difficult. The auto industry is pursuing better quality from the beginning. For massive produced parts such as the transmission valve body, fast (within the production cycle time of less than a few minutes per part) and precise in-line inspection is required. Currently there is no in-line automated measurement technique available for porosity inspection in small bores. Industrial borescopes (manufactured by companies such as GE, Olympus, Hawkeye, etc) are the only available tools which are widely used for manual vision inspection in most factories. Traditional visual inspection requires not only operator skills to manipulate the whole procedure properly but also experience to interpret the measurement results. However, none of these borescopes can perform rapid and accurate measurement in-line, which is desired by the industry.

Non-contact measurement techniques were employed for quality control [1-4]. Among them image processing and machine vision have been highly improved in the past 10 years because of the rapid development and maturing of 
semiconductor and computer software techniques. They provide accurate nondestructive measurements [5, 6]. The speed and precision of machine vision measurement opened a path for in-line or in-process inspection that could improve the repeatability of measurements. A reconfigurable inspection machine (RIM) was designed based on machine vision and laser techniques to inspect $100 \%$ a family of engine cylinder heads. It detected key features with accuracy in tens of microns in-line [7-10].

Current machine vision systems work well in imaging flat surfaces in most cases. To inspect a curved or complex surface, challenges are introduced to the illumination and optics (lens, light source, light guide) design. This paper presents a methodology for detecting surface defects such as pores inside small bores using a machine vision approach. The system utilizes an innovative $360^{\circ}$-view line-scan algorithm combined with a novel coaxial surface illumination technique to acquire high-quality, high-definition imagery of the inner surface of small bores. Image processing algorithms are utilized to detect, classify and precisely locate the pores. Geometrical filters allow the end user to choose the pore characteristics for which they wish to search. The scanning movement and image acquisition were synchronized to guarantee the fidelity of the image reconstruction. A prototype measurement machine to demonstrate the concept of in-line inspection of small bores was built by the Engineering Research Center (ERC) at the University of Michigan to measure the small bores in transmission valve bodies. These step bores have a diameter measuring from $8 \mathrm{~mm}$ to $15 \mathrm{~mm}$ and have a complicated inner surface. The experimental results show that the prototype system can correctly identify pores over $500 \mu \mathrm{m}$ within these bores.

\section{Overall approach}

\subsection{Basic principle}

To perform an accurate image-based measurement, the first step is to acquire high-quality, high-definition imagery of the surface of the internal bore. Special designed optics combined with a novel line-scan method fulfills the image acquisition precisely. Line scanning $[11,12]$ is a mature technique widely used in industry and medical examination. The image reconstruction of a line scan is as simple as stitching the straight line strips together in sequence of scanning. This technique has been proven to be adequate for imaging a flat target.

A conical lens is an optical device which can image a $360^{\circ}$ view of the inner surface of a bore. It works like a periscope bending light $90^{\circ}$ from its incidence angle. The system's optical image magnification stays constant in an annular zone which is concentric with the conical center. This annular zone can be used as a line similarly to the line scan. By unwrapping the image of the annular zone to a straight line and stitching these lines together in the sequence of scanning, a flat image of the inner surface of small bores can be reconstructed. This basic image reconstruction flow is explained in figure 1.

The sight-pipe with conical lens at the tip was utilized in the ERC prototype to perform this novel scanning

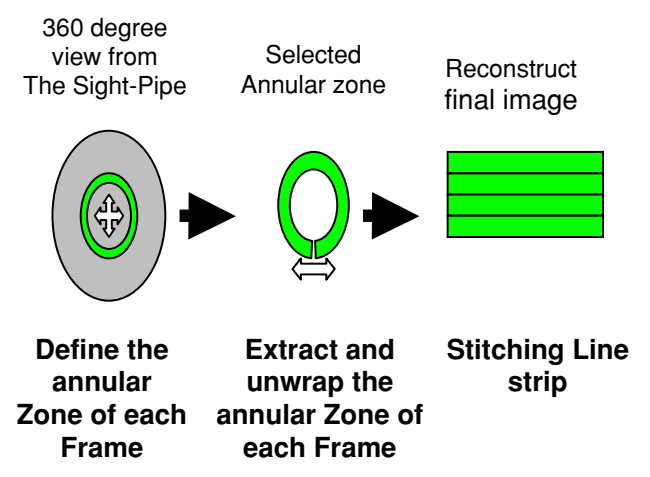

Figure 1. Image reconstruction flow.

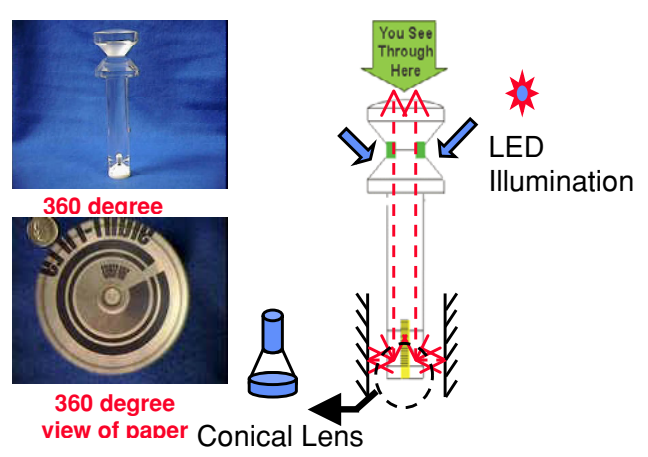

Figure 2. Optical principle of sight-pipe.

methodology. It guides the illumination as well as imaging the surface of small bores. Specially designed LED coaxial illumination precisely matched with the sightpipe's illumination surface increased the efficiency of the illumination, and also overcame the dimensional limitations of the small bore. Figure 2 shows the optical principle of a sight-pipe. After the image acquisition and reconstruction, an automatic threshold decision algorithm is introduced to find the pores specified by the part requirements.

\subsection{The implementation}

The prototype small bore porosity inspection system was developed and implemented by the ERC as shown in figure 3. Three Aerotech linear motion stages are used: two of them ( $X$ - and $Y$-axis) adjust the position between part/fixture and the inspection system and third one ( $Z$-axis) performs the scanning. Two three-dimensional tilt stages were used to align the axis of motion, optical axis and bore axis. One objective was to identify the alignment requirement among the axes for acquiring a clear image of the inner surface of small bores. The machine vision subsystem utilized to acquire the images includes a Pulnix $\frac{1}{2}$ inch CCD camera with $1392 \times$ 1045 pixels, Edmund Optics close focus lens, a CCS Dome LED light and three sight-pipes $(6 \mathrm{~mm}, 10 \mathrm{~mm}, 13.3 \mathrm{~mm}$ diameter) with exchangeable mounting collar and other related mechanical mounting parts. The assembled system is shown in figure 4.

Labview was the sole language used in this research to fulfill all of the system control, data acquisition and data analysis. 


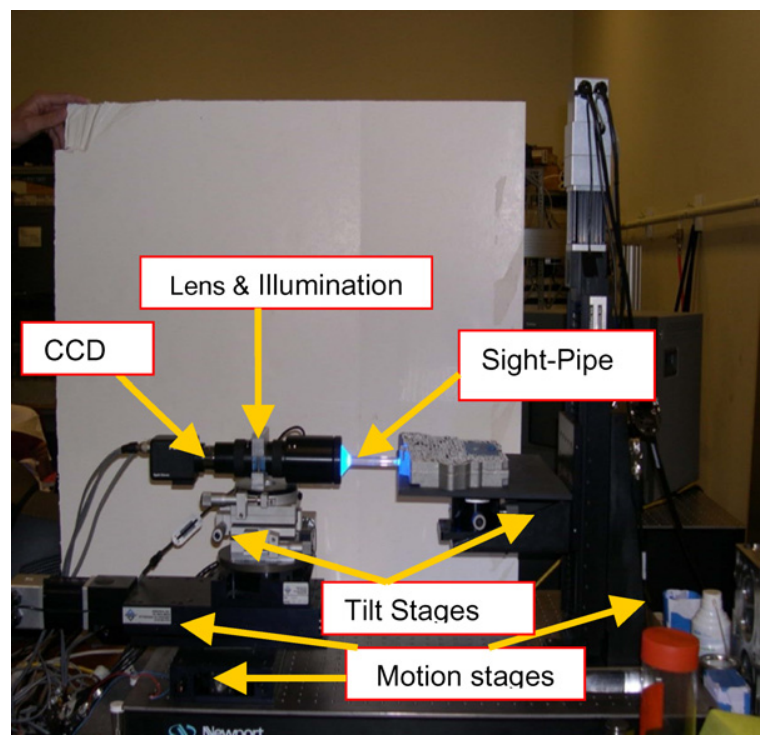

Figure 3. Small bore inspection system.

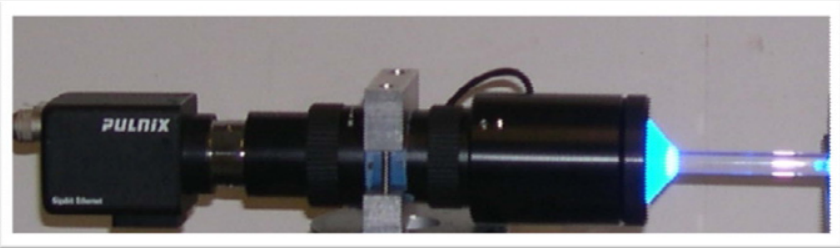

Figure 4. Submachine-vision system assembly.

\section{Challenge and solutions}

Evaluation of the porosity inspection system was carried out in several steps.

(1) Basic concept validation. This study evaluated the ability of the system to reconstruct an image without geometrical distortion. Multiple printed papers with designed patterns were wrapped into small bores for scanning and studying. The results of this study confirmed the feasibility of the original concept.

(2) System performance study on real parts. It evaluated the ability of the system to detect pores in parts that were made intentionally where the porosity exceeded the allowable specification. The surface porosity was generated by drilling small holes on the bore surface with specified diameters. The complicated real inner surface of transmission parts introduced great challenges for the system calibration and pore analysis algorithms. An experimentally achievable calibration procedure was proposed and a self-adaptive pore analysis algorithm was developed to overcome the extreme intensity variance introduced by parts and system misalignment.

(3) Extensive study. Different wavelength illuminators were studied to achieve the best image contrast. Machining leftover marks, which existed during in-line measurement, were also studied. Repeatability results were obtained using 50 independent test runs performed in a single bore.

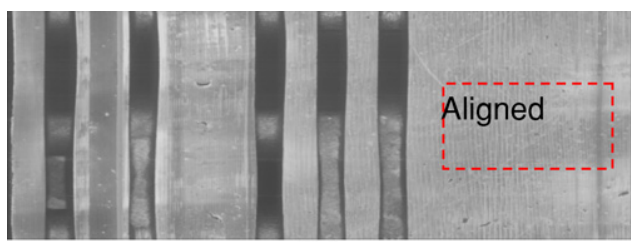

(a)

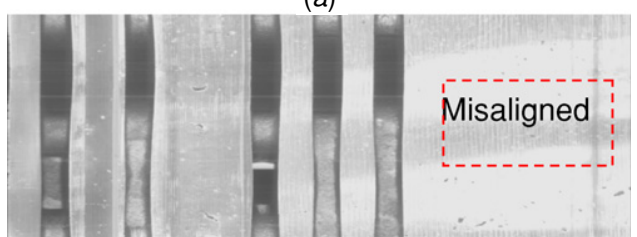

(b)

Figure 5. Difference between good alignment and misalignment: (a) well aligned, $(b)$ misaligned with black and white strips.

\subsection{Challenges}

The above studies disclosed that a successful small bore inspection based on image measurement has to overcome the following challenges.

(1) Optical alignment of the motion stage, including the image system and bore axis alignment, was found essential for scanning. Misalignment can cause a decrease in image quality, distortion from the target image, misrecognitions in pore analysis, and could cause damage to the optical components. The accuracy required for proper system alignment, such as concentricity and angular error, was quantified through experimental calibrations. An efficient procedure was developed to perform the proper alignment.

(2) The accuracy of the synchronization among the motion and image acquisition components determined the final image reconstruction and system's performance. The triggering technique drastically reduced overlapping or missing data caused by the velocity variance of the motion stage.

(3) The PC memory involved in image acquisition had to be managed efficiently. Otherwise, the massive data acquired through the area CCD scanning could be limited by the bottleneck of memory in the operating system.

(4) The requirements of the in-line measurement system were for the total processing time to be lower than the machining cycle time. New multi-core programming techniques were introduced in this research and expedited the system performance.

(5) The optical calibration determined the precision of the measurement, which was important for a machine vision system. A series of optical test procedures were designed for calibration purposes. Without these specially designed methods it was extremely difficult to make a high-quality measurement.

\subsection{Solutions}

3.2.1. Optical alignment. Because of coaxial illumination used in the system, the misalignment of the optical axis significantly affects imaging quality. Figure 5 shows a 
significant difference in standard deviation (STD) between alignment and misalignment. Issues of misalignment cause errors in the accuracy of the final system; therefore, a proper alignment procedure must be developed. There were two criteria to consider for optical alignment of the system: the sight-pipe and the bore to be scanned must be coaxial, and the direction of motion must be parallel to the axis of the sightpipe. When the axes were not aligned, both non-uniform lighting and image distortions were introduced to the system, resulting in errors such as misdetections and incorrect pore size analysis.

In order to quantify the precision of alignment needed for the system, a series of tests were performed. The experiment involved performing multiple scans at different levels of misalignment. The results indicated that the system was accurate and repeatable if all axes were aligned within $100 \mu \mathrm{m}$ for a maximum $150 \mathrm{~mm}$ scanning depth among 8-15 mm diameter bores, including linear and angular alignments estimated by equation (1):

$$
x+D \times \tan \theta \leqslant 100 \mu \mathrm{m}
$$

where $x$ is the linear radial offset, $D$ is the length of scan and $\theta$ is the angular offset.

An alignment procedure was developed in order to reduce the size of errors caused by misalignments. The procedure may be broken up into two parts: one to align the direction of motion with the sight-pipe, and the other to align the sight-pipe with the bore. The procedure is explained by the following steps.

(1) Align the sight-pipe with direction of motion based on a method using geometry.

(a) Using a reference circle with a known diameter, remove the sight-pipe and place the circle near the camera lens system. Make note of the diameter and center of the circle.

(b) Move the motion stage system back to a known distance and adjust the magnification of the system to get the same diameter as in the close position.

(c) From the distance that the center of the circle has traveled and the distance the motion stage has traveled, the angular misalignment of the optical system, and therefore the pitch and yaw of the sight-pipe, related to the motion axis was calculated using equation (2) and then corrected as illustrated in figure 6:

$$
\theta=\sin ^{-1} \frac{\Delta x}{D} .
$$

(2) Align the bore with the sight-pipe based on a method for through holes. The method required two clear circular edges within the bore: one at the opening (at the entrance) and the second near the rear (or the exit for through bores). This was available for through bores and in spool bores with several lands. Bore axis was defined by connecting the two circular center points. The method involves the following steps.

(a) With the sight-pipe on and adjusted to the correct magnification settings, find the center of the circular plug (figure 7).

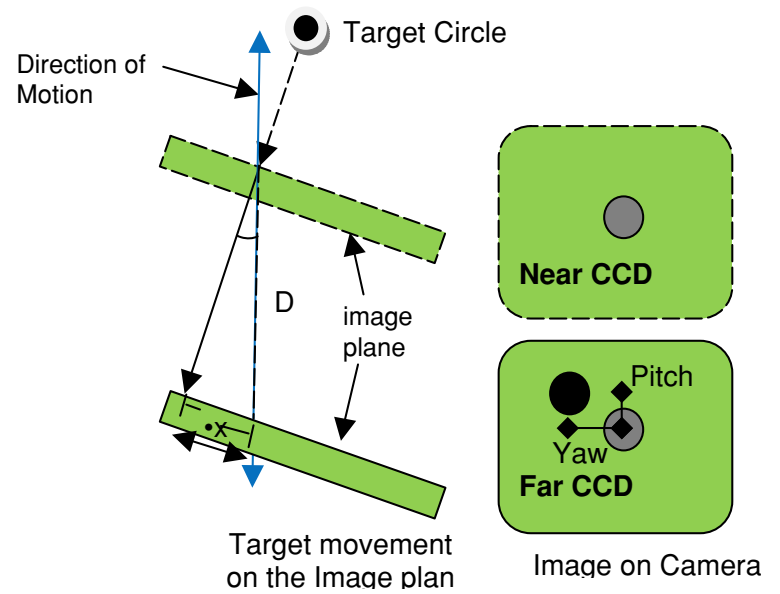

Figure 6. Alignment of sight-pipe with direction of motion.

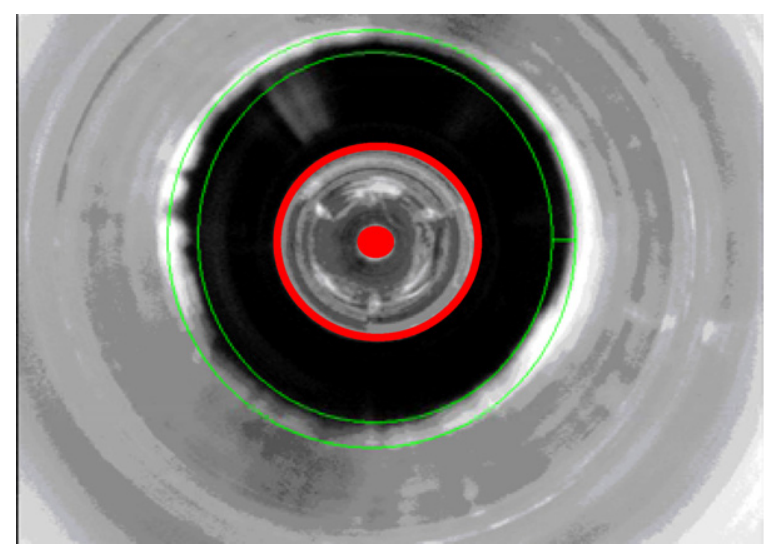

Figure 7. Finding the center of the sight-pipe.
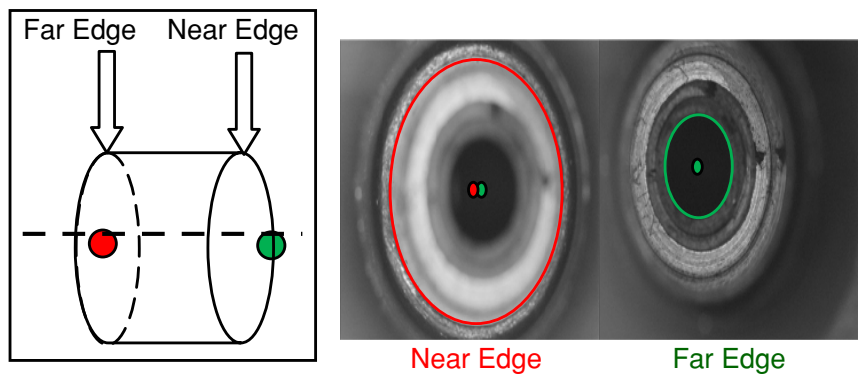

Figure 8. Shows focusing positions used to align bore axis with sight-pipe axis.

(b) Remove the sight-pipe and focus on the opening of the bore so that you can see the edge clearly and find the center (figure 8, left).

(c) Next focus on a circular edge near the end of the bore and find the center (figure 8, right).

(d) Adjust the pitch and yaw of the part until the centers of these circles coincide.

(e) After pitch and yaw are aligned, adjust the linear position of the part until the center of the circles coincides with the previously found center of the sight-pipe.

The method described above worked well for many of the bore samples that were scanned in the lab environment. 


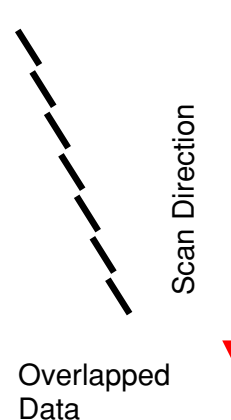

Data

Figure 9. Demonstration of overlapped and missing data caused by miscalibrated image capture.

However, there are some that do not have clear edges to focus on.

\subsubsection{Synchronization of image capture with the motion stage.} The image acquisition needed to be synchronized with the motion in order to record the proper measurements along the axial direction. If the image capture was too slow or too fast, there would be some overlapping or missing data respectively as illustrated in figure 9. Such incorrect data could cause incorrect pore size and location measurements.

Two different methods were used in calibrating the image capture: (1) velocity-based and (2) position-based acquisitions. When the velocity-based acquisition was utilized, the frame rate of the camera was set to a constant that was based on the speed of the motion stage and the width of the region of interest given by equation (3):

$$
\text { Frame rate }(\mathrm{fps})=\frac{\text { Motion stage's velocity }\left(\frac{\mathrm{mm}}{\mathrm{s}}\right)}{\text { Width of annular zone }\left(\frac{\mathrm{mm}}{\mathrm{frame}}\right)} \text {. }
$$

The velocity variance of the motion stage caused the images to overlap in some regions and miss data in other regions. To provide a system with greater accuracy, position-based triggering was used and it was sent from the motion stage. Precision motion control applications often required triggering an external device based on the actual position of the axis in motion. For many in-line high-speed-measuring applications such as image scanning, traditional methods of triggering compromised the precision and cycle time of the measurement. A unique high-speed position-based trigger called the position synchronization output (PSO) [13] provided benefits to these applications. Enabled by a combination of hardware, software and advanced algorithms, the trigger allowed starting, stopping or pulsing the optical sensor to take readings on the fly from measuring probes or cameras. In fact, any application that required precise data acquisition linked to axis position could benefit from PSO functionality. The key advantage of PSO was its ability to trigger the output based on actual axis position in the submicron precision range which eliminated the external disturbance introduced in the process. For example, the velocity regulation and settle value became unimportant. Also the latency between axes reached the actual position and the triggering output was below a microsecond. This allowed high-speed measurement with much less overlapping
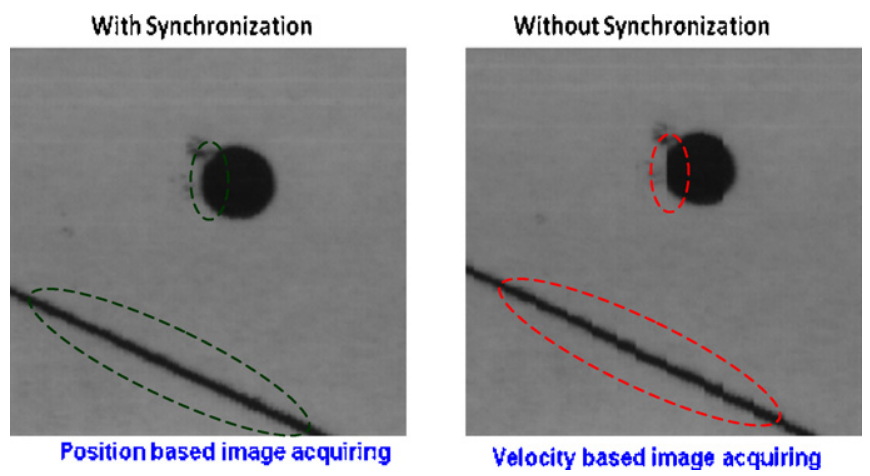

Figure 10. PSO image acquisition versus velocity image acquisition.

and fewer missed events compared to traditional high latency tracking methods. Another significant pro of PSO was its high-frequency pulsed trigger which could operate at $10 \mathrm{MHz}$ and totally satisfied many laser-based high-speed data acquisitions. The introduction of PSO into the small bore inspection system produced final images with much less overlapping and fewer missed frames and higher measurement precision. It is clearly shown in the circled area of figure 10 .

3.2.3. Memory management. The massive amounts of memory acquired during a scan were managed to prevent bottlenecking by the memory of the operating system. The images were monitored during a scan and the memory was released as soon as processing was done. The Pulnix CCD camera that was used in the current system had $1392 \times$ 1045 pixels with 8 bit image depth per pixel; with the availability of $3 \mathrm{~GB}$ of maximum processing RAM of a 32 bit Windows system, while the program was running, we could only acquire 257 frames without using correct memory management. The following equation (4) was used to calculate the number of frames available with memory:

$$
\frac{\text { RAM available }}{\text { Pixel area } \times 8 \mathrm{bpp}}=\frac{3 \times 10^{9}}{1392 \times 1045 \times 8 \mathrm{bpp}} \approx 257 \text { frames. }
$$

For example, the longest scan needed in the valve bodies was $150 \mathrm{~mm}$, with $0.144 \mathrm{~mm} /$ frame, a minimum frame count of 1041 frames is required. This did not lie within the available memory specified above which would lead to a bottleneck condition for the program.

In order to manage all aspects of memory problems in the current system, it was required to use as little memory as possible during a scan, and to release memory as soon as the data were analyzed and had no further use. To cut back on memory during a scan, the selective scan area available with the Pulnix CCD camera along with a cropping function were used to keep the output area as small as possible. This increased the number of frames available from 257 to 1092 , which allowed the user to acquire the number of frames needed for a $150 \mathrm{~mm}$ scan. Memory was also released from the program as soon as it was done being processed to allow other processes within the program to use the memory. 
3.2.4. Time constraints of in-line measurements. Currently the cycle time of the current system to scan one $100 \mathrm{~mm}$ bore was approximately $20 \mathrm{~s}$ with an annulus width of 6 pixels. In the small bore porosity inspection system, the image acquisition took up the majority of the cycle time. Although the acquisition speed was limited by the parameters of our CCD camera, there were multi-core programming techniques available to do post-processing during an acquisition. This method, called the producer/consumer method, used dual core technology and queues in LabVIEW to process data as they were acquired. One core was acquiring all of the data from the acquisition. When an optimal amount of information was acquired, the data were pushed into a queue. These data were then immediately available to a separate section of code that was unwrapping and pasting the information together on the second core. By implementing this producer/consumer programming into our system, we were able to knock off $10 \%$ of the cycle time of our system.

There were two methods available to us that can speed up the acquisition speed: (1) an increase in the sampling annulus width, and (2) an upgrade to a faster camera (CCDs with faster frame rates). A larger sampling annulus would allow a greater axial length of the bore to be acquired for each frame, giving a decrease in time. This could reduce the acquisition of a $100 \mathrm{~mm}$ scan from $20 \mathrm{~s}$ to around $8 \mathrm{~s}$ by using a width of 18 pixels in the current system. The drawback of this approach was that the larger the annulus, the greater the distortion caused by unwrapping each frame. This distortion caused the final image to be blurred and hard to process. A faster camera could speed up the system by enabling the capture of more frames per second.

\section{Test results}

The repeatability of the system was tested to validate the system performance. Drilled holes with known size were used to simulate real pores in this test (see figure 11). Additional holes were drilled between the lands, but they should not be detected because they were masked. The software and hardware were restarted and reinitiated before every test. The pore size (using the minimum circumscribed pore diameter), pore area and location were estimated through the above-described image processing technique. The aim of this test was to quantify the system repeatability including the positioning motion stage system, the optical acquisition, the image analysis module and the overall software controlling the system. Test part variance and operator-induced variance were not in the scope of the test. As such the test was run by a single operator using a single part and a designated bore in the part. The test was repeated 50 times and the results were collected for each run. Software and hardware were reinitialized before each run.

The experimental setting process is as follows.

(1) The part was properly located on the fixture using dowels. The optical system was aligned manually at the beginning, and maintained constant throughout. This alignment includes the pitch and yaw adjustments of the optical system as well as the magnification settings of the lens.

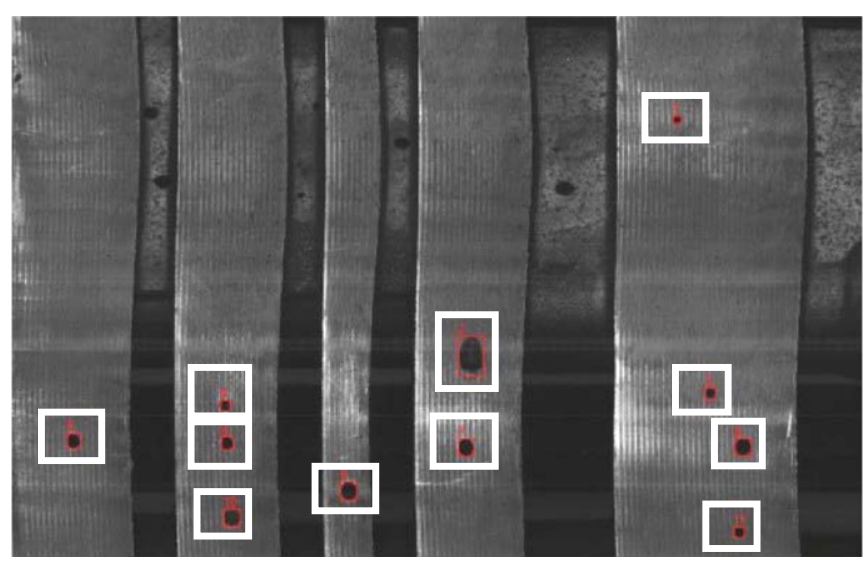

Figure 11. Illustration of the image used in the repeatability test.

5.15 Sigma Variation as a Percent of Actual Pore Size

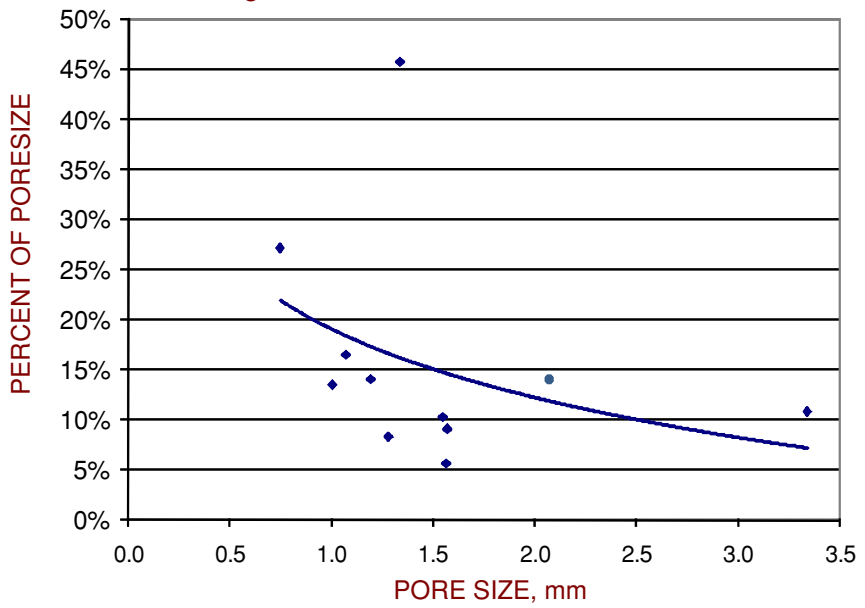

Figure 12. Sigma variations as a percentage of pore size.

(2) The blue light coaxial illumination was maintained $\mathrm{ON}$ at all times during the test.

(3) The scanning parameters, including the starting coordinates, the scan depth and the scan rate, were constant throughout the analysis. The homing position of the axes is fixed.

(4) The pore detection filter parameters were maintained constant throughout the duration of the analysis.

The tested bore's diameter was $12.8 \mathrm{~mm}$ and the scanning depth was $75 \mathrm{~mm}$. The total scanning and pore detection cycle time was approximately $18 \mathrm{~s}$. The bore diameter and its position were calculated from the image processing algorithm developed for this project. Final images are shown in figure 11 .

The 5.15 sigma variation as a percentage of actual pore size was estimated for the 11 pores. The actual pore size was calculated as the average value of the 50 measurements. The distribution is shown in figure 12 as a function of pore size. Most of the results are below 20-30\% variation. A similar analysis was made using the area of the pores and the results are given in figure 13. Most of the pores were below 35\%. It 
5.15 Sigma Variation as a Percent of Actual Pore Area

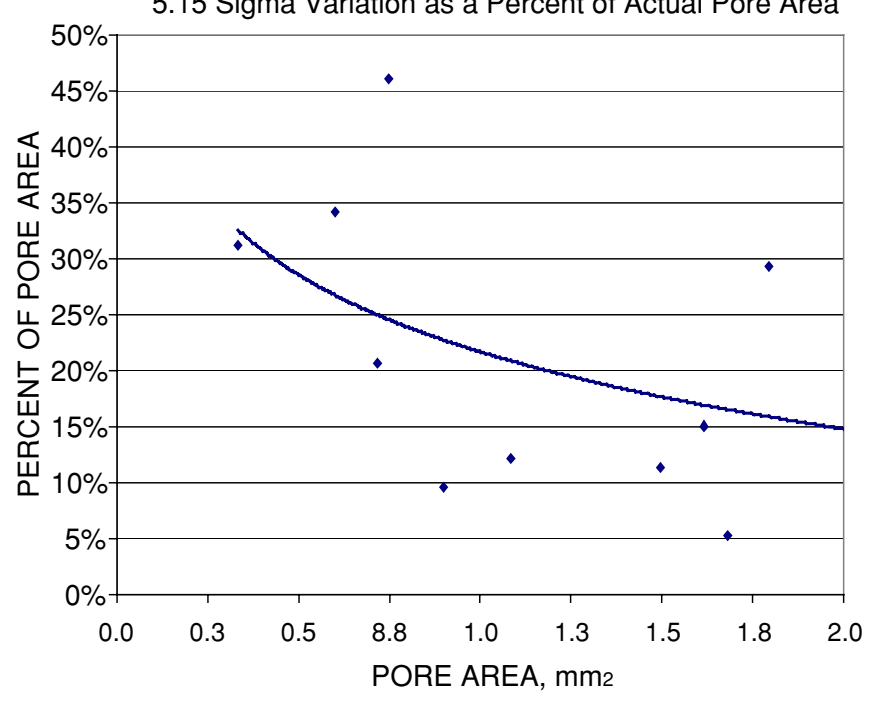

Figure 13. Sigma variations as a percent of pore area.

seemed that the pores with the smaller area resulted in a higher percentage of pore area.

The evaluation of the pore location required a reference value of greater than $0.7 \mathrm{~mm}$ and for some of the pores as high as $1.5 \mathrm{~mm}$ in order to pass the test. The criterion of the gage potential index is $C_{\mathrm{g}} \geqslant 1.55$. RF is a tolerance defined as the upper specification limit (USL) minus the lower specification limit (LSL). For this study RF was taken to be in the range of $0.7-1.5 \mathrm{~mm}$ depending on the pore size variation. The gage potential is defined as

$$
C_{\mathrm{g}}=\frac{0.2 \mathrm{RF}}{6 S_{\mathrm{g}}}
$$

where $S_{\mathrm{g}}$ is the standard deviation of measurements of the gage system.

The sight-pipe alignment requirement for the bore axis was evaluated by performing the bore porosity analysis at different misalignments from 0.1 to $0.8 \mathrm{~mm}$. The results illustrated that a misalignment of $0.1 \mathrm{~mm}$ in $100 \mathrm{~mm}$ probe scan length had no effect in identifying porosity. However, as the alignment increased to $0.2 \mathrm{~mm}$, higher false detection of pores became apparent. Therefore, the misalignment can be specified safely as $0.001 \mathrm{~mm} \mathrm{~mm}^{-1}$ or about 205 arc seconds.

The depth of field for the sight-pipe was evaluated because it affected its agility and cycle time when it is used in parts with multiple bores such as the valve body. The evaluation of the three sight-pipes indicated that each can measure a bore with a size range up to $80 \%$ or $100 \%$ larger than its size. A list of standard deviations of all parameters over the runs is presented in table 1 .

The average positioning precision of the pore (6 sigma) is under $18 \mu \mathrm{m}$ and the standard deviation of the measured equivalent pore diameter (6 sigma) is under $190 \mu \mathrm{m}$. This proves that the system has the capability to detect pores above $500 \mu \mathrm{m}$.
Table 1. Measurement results—standard deviation.

\begin{tabular}{llll}
\hline $\begin{array}{l}\text { Pore } \\
\text { number }\end{array}$ & $\begin{array}{l}\text { Center of } \\
\text { mass } X(\mathrm{~mm})\end{array}$ & $\begin{array}{l}\text { Center of } \\
\text { mass } Y(\mathrm{~mm})\end{array}$ & $\begin{array}{l}\text { Diameter } \\
(\mathrm{mm})\end{array}$ \\
\hline 1 & 0.014 & 0.023 & 0.039 \\
2 & 0.008 & 0.015 & 0.066 \\
3 & 0.014 & 0.039 & 0.021 \\
4 & 0.014 & 0.018 & 0.025 \\
5 & 0.011 & 0.020 & 0.018 \\
6 & 0.011 & 0.018 & 0.024 \\
7 & 0.016 & 0.020 & 0.031 \\
8 & 0.009 & 0.018 & 0.031 \\
9 & 0.038 & 0.035 & 0.041 \\
10 & 0.011 & 0.018 & 0.015 \\
11 & 0.016 & 0.016 & 0.034 \\
\hline
\end{tabular}

\section{Conclusion}

It has been shown that the small bore inspection probe can be used to identify porosity defects that have a diameter of greater than $0.5 \mathrm{~mm}$ in small bores. The probe system measurements were satisfactorily repeatable based on studies of the percentage of pore size and area.

The full procedure for the porosity inspection system was developed and evaluated. The prototype of the system was built and tested using the proposed methodology. Results have shown that an accurate image can be reconstructed using principles of line scanning along with the unwrapping algorithms provided by LabVIEW. The proposed system can reconstruct the inner surfaces of bores and detect pores that have a diameter of greater than $0.5 \mathrm{~mm}$. The pore location was identified with an average location tolerance of $0.7 \mathrm{~mm}$ range and in some cases the maximum of $1.5 \mathrm{~mm}$ range. The alignment precision required between the sight-pipe and the bore was about $100 \mu \mathrm{m}$ for $100 \mathrm{~mm}$ scan inside a bore. The depth of field was determined to be $40 \%$ and $60 \%$ of the sightpipe's diameter, respectively, for the $6-10 \mathrm{~mm}$ and $13.3 \mathrm{~mm}$ sight-pipes. This indicated that one sight-pipe could be used for a range of bores within twice its diameter. Selection of the light source wavelength, such as red versus blue, did not provide any advantages.

\section{Acknowledgments}

The authors gratefully acknowledge the financial support of the Engineering Research Center for Reconfigurable Manufacturing Systems at the University of Michigan. They would also like to thank $\mathrm{Mr}$ Michael Swanger for his suggestions throughout the execution of the method and his continuous technical support. In addition, they would like to extend their appreciation to Steve Erskine and Scott Hyder of the ERC for their assistance in creating the fixture parts used in this study. Finally, they would like to thank Aerotech for their donation of a motion stage used in this research.

\section{References}

[1] Iborra T, Alvarez B, Jimenez C, Fernandez Meroño J M, Fernández C and Suandiaz J 1999 Automated visual 
inspection (AVI) system for crankshaft production processes PHOTOMEC'99/ETE'99 (November 1999, Liège)

[2] Iborra T, Fernandez Meroño J M, Fernández C and Suandiaz J 1999 On-line automated visual inspection for quality control within the automobile industry Proc. QCAV99-5th Int. Conf. on Quality Control by Artificial Vision (May 1999, Quebec, Canada)

[3] Broggiato G B, Campana F and Gerbino S 2001 Shape deviation analysis on sheet-metal parts through reverse engineering techniques XII ADM Int. Conf. on Design Tools and Methods in Industrial Engineering (5-7 September 2001, Rimini, Italy)

[4] Lipshitz B and Fischer A 2003 Verification of scanned engineering parts with CAD models based on discrete curvature estimation CIRP 2nd Int. Conf. on Reconfigurable Manufacturing (20-21 August 2003, Ann Arbor, MI)

García D, Sebastián J M, Sánchez F M, Jiménez L M and González J M 1999 3D inspection system for manufactured machine parts Proc. SPIE 3652 250-60

[5] Blais F 2003 A review of 20 years of range sensor development Proc. SPIE 5013 62-76

[6] Fraser C 1999 Automated vision metrology: a mature technology for industrial inspection and engineering surveys 6th South East Asian Surveyors Congress (1-6 November 1999, Fremantle, WA)

[7] Katz R, Zuteck M G and Koren Y 2002 Reconfigurable inspection machine for machining production lines $G P C$ Proc. 23 228-35

[8] Katz R, Zuteck M G and Koren Y 2002 Rapid inspection and error tracing methodology for machining production lines 3rd CIRP Int. Seminar on Intelligent Computation in Manufacturing Engineering-ICME (3-5 July 2002, Ischia, Italy)

[9] Koren Y and Katz R 2003 Reconfigurable apparatus and method for inspection during a manufacturing process $U S$ Patent No 6,567,162 B2

[10] Barhak J and Katz R 2004 Rapid non-contact measurements of engine cylinder heads with the reconfigurable inspection machine 4th CIRP Int. Seminar on Intelligent Computation in Manufacturing Engineering-ICME' 04 (30 June-2 July 2004, Sorrento, Italy)

[11] Yao M H and Sharp C 1996 Image processing for a line-scan camera system 30th Asilomar Conf. on Signal, System and Computers (November 1996, CA)

[12] Hon H W and Evans J P O 2002 Multiple-view line-scan imaging IEE Proc. Optoelectron. 149 45-50

[13] Cox B 2006 Precise triggering of external events based on axis position Design News July 